\section{Endoscopic Image of a Splenic Artery Aneurysm}

A 66-year-old woman with a history of five pregnancies and mild arterial hypertension presented with symptomatic gallstone disease and sonographically documented calcifications near the splenic hilum. Upper endoscopy revealed a hemispheric tumor $4 \mathrm{~cm}$ in diam

eter in the upper part of the back wall of the stomach (Figure 1). The overlying mucosa was intact (Figure 2). Endoscopic ultrasonography was not yet available, and the finding was interpreted as a leiomyoma of the stomach wall. As endoscopic biopsy of such lesions is usually inconclusive, it was not performed. A barium meal study confirmed the presence of an intramural mass.

In addition to cholecystectomy, therefore, enuclation of the tumor was recommended in order to eliminate a potential bleeding source or malignancy. Intraoperatively, the small sac was explored and a calcified aneurysm of the splenic artery $4 \mathrm{~cm}$ in diameter was found. It was pushing the normal-appearing stomach wall towards the luminal face, simulating an intramural tumor. The aneurysm was resected. The clinical diagnosis was confirmed by histopathological examination (Figure 3 ). The patient had a smooth recovery and is currently well.

Splenic artery aneurysmal disease is rare. It occurs more often in women $(87 \%)$ and increases with the number of pregnancies. The main etiological factor is arterial medial degeneration during pregnancy (1). The rate of rupture is about $3 \%$ (2). Symptomatic aneurysms, aneurysms increasing in size or exceeding $2 \mathrm{~cm}$ in diameter, as well as aneurysms discovered during pregnancy, should be resected (2).

Though most patients with splenic artery aneurysms are asymptomatic, some complain of nonspecific upper abdominal pain. If a bulging of the upper posterior wall of the stomach is found endoscopically, a splenic artery aneurysm should be considered. Nowadays, endoscopic ultrasonography should be used to evaluate the nature of masses protruding into hollow organs. It allows exact determination of all layers of the intestinal wall and of adjacent organs, and may be superior to standard investigations.

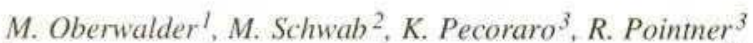

${ }^{1}$ IInd Department of Surgery, University of Innsbruck, ${ }^{2}$ Department of Internal Medicine, ${ }^{3}$ Department of Surgery, A.ö. Krankenhaus Zell am See, Austria

\section{References}

1. Stanley J, Thompson NW, Fry WJ: Splanchnic artery aneurysms. Arch Surg 1970; 101: 689-697.

2. Trastek VF. Pairolero PC. Bernatz PE: Splenic artery aneurysm. World J Surg 1985; 9: 387-383.

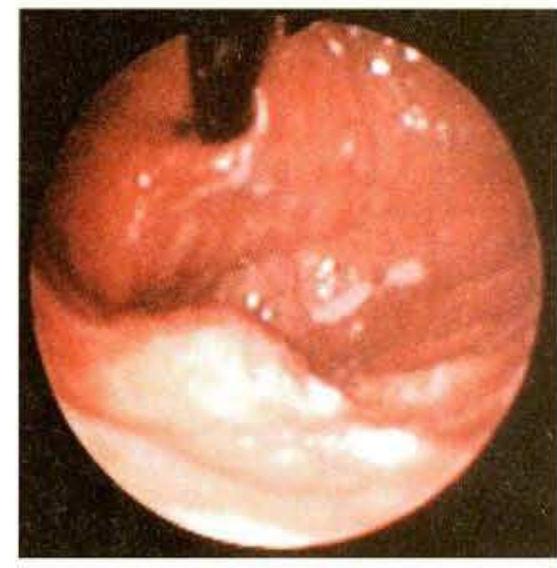

Figure 1: The endoscope in inverted position. Bulging of the posterior wall of the stomach.

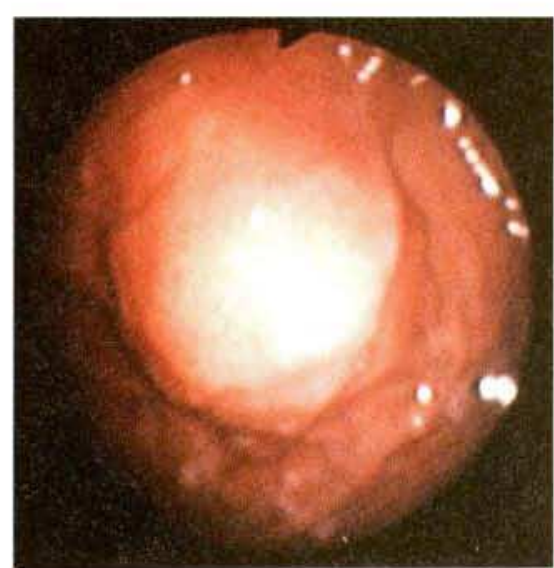

Figure 2: Direct view of the mass.

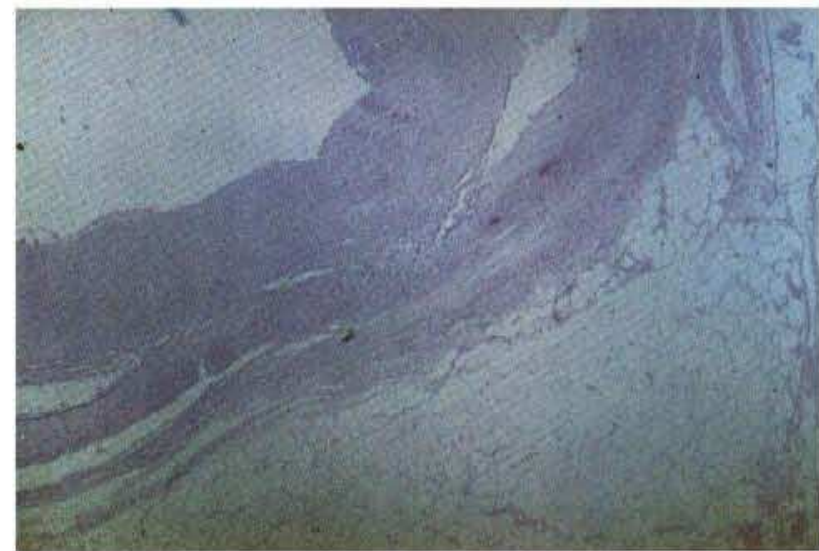

Figure 3: Histopathological specimen: true aneurysm of the splenic artery. 